\title{
Atypical Familial Mediterranean Fever in a Japanese Boy with Heterozygous MEFV p.Ser503Cys Exon 5 Variant
}

\author{
Tomonobu Sato ${ }^{1},{ }^{1}$ Shunichiro Takezaki, ${ }^{2}$ Takeru Goto, ${ }^{1,2}$ Shinichi Ishikawa, ${ }^{1}$ \\ Kazumi Oura, ${ }^{1}$ Asuka Takahata, ${ }^{1}$ Haruki Shiraishi, ${ }^{1}$ Yuji Maruo, ${ }^{1}$ Norio Sato, ${ }^{1}$ \\ Takashi Suganuma, ${ }^{1}$ and Makoto Mikawa ${ }^{1}$ \\ ${ }^{1}$ Department of Pediatrics, Kitami Red Cross Hospital, Kitami, Japan \\ ${ }^{2}$ Department of Pediatrics, Hokkaido University Hospital, Hokkaido, Japan \\ Correspondence should be addressed to Tomonobu Sato; sato-tkyn201904@wave.plala.or.jp
}

Received 11 November 2020; Revised 8 February 2021; Accepted 16 February 2021; Published 2 March 2021

Academic Editor: Carmelo Romeo

Copyright (c) 2021 Tomonobu Sato et al. This is an open access article distributed under the Creative Commons Attribution License, which permits unrestricted use, distribution, and reproduction in any medium, provided the original work is properly cited.

\begin{abstract}
Periodic fever syndromes are heterogeneous diseases. Familial Mediterranean fever (FMF) is one of the hereditary periodic fever diseases caused by a Mediterranean fever $(M E F V)$ gene abnormality. FMF can be categorized as typical or atypical, based on clinical findings and genetic screening. Atypical FMF has a wide variation of clinical findings and disease-causing mutations of $M E F V$. Therefore, it is sometimes difficult to diagnose an unknown fever as FMF. To date, a large number of various typical and atypical FMF cases have been reported in Japan. Here, we describe a Japanese boy with heterozygous MEFV p.Ser503Cys exon 5 variant who developed periodic fever. He was treated with colchicine; a complete eradication of his fever and various accompanying symptoms have been subsequently achieved for more than a year. Given that there have been a few reports about patients with this variant, little is known about the genetic and phenotypic role of heterozygous MEFV p.Ser503Cys exon 5 variant. It is therefore imperative to consider atypical FMF as a differential diagnosis when a periodic fever is encountered. Furthermore, we suggest that it is worthwhile to integrate $M E F V$ gene analysis with the potential effects of colchicine treatment in patients with periodic fever.
\end{abstract}

\section{Introduction}

Periodic fever syndromes are clinically characterized by recurrent episodes of fever, systemic inflammation, and symptoms such as skin rashes, abdominal pain, chest pain, lymphadenopathy, or arthritis [1]. Familial Mediterranean fever (FMF) is one of the hereditary periodic fever diseases associated with an abnormality in the Mediterranean fever $(M E F V)$ gene, which induces dysfunction of the inflammasome, followed by the production of interleukin- $1 \beta$. In particular, FMF is caused by a dysfunction in pyrin, which is encoded by the $M E F V$ gene [2]. FMF may be typical or atypical, depending on clinical findings and genetic screening [3]. The clinical characteristics of typical FMF are periodic, self-limiting fever lasting three days at most accompanied by serositis, pleuritis, peritonitis, and arthritis.
FMF is considered an autosomal recessive disease, and homogeneous or compound heterozygous $M E F V$ mutations in exon 10 are correlated with a typical FMF phenotype [3]. In contrast, patients with atypical FMF develop symptoms that do not necessarily match those of typical FMF and harbor variants or heterozygous $M E F V$ mutations, different from those of typical FMF. In atypical FMF cases, bouts of fever, for example, last for more than four days, attacks of serositis present as localized, and nonspecific symptoms, such as arthralgia and myalgia, are observed [4]. There are no specific hematological markers for atypical FMF; therefore, diagnosis and initiation of treatment are often delayed [5]. To date, a large number of FMF variants cases have been reported in Japan [6].

Here, we report the case of a Japanese boy with heterozygous MEFV p.Ser503Cys exon 5 variant 
(NM_000243.2(MEFV): c.1508C > G; p.Ser503Cys) with periodic fever, improved by treatment with colchicine.

\section{Case Presentation}

An 11-year-old Japanese boy developed a prolonged fever lasting 16 days, general malaise, abdominal pain, and slight headache prior to admission. He revealed no significant chest pain or arthralgia. His medical history was unremarkable. He was diagnosed as having upper respiratory tract infection by his primary care physician and was treated with nonsteroidal anti-inflammatory drugs, antibiotics (tosufloxacin), and steroids (prednisolone, $1 \mathrm{mg} / \mathrm{kg} /$ day for 5 days). However, his prolonged fever was not eradicated, and he was referred to our department and was admitted to our ward. On admission, he was alert and oriented. He had no special family history of FMF and was not a child of a consanguineous marriage. Physical examination was unremarkable except for a body temperature of $38.4^{\circ} \mathrm{C}$ and aphthous stomatitis. He revealed no meningeal signs. Hematological tests were normal; blood biochemistry tests revealed the presence of high acute reactants (C-reactive protein, CRP: $3.30 \mathrm{mg} / \mathrm{dL}$; serum amyloid A protein, SAA: $920.0 \mu \mathrm{g} / \mathrm{mL}$ ) and elevated erythrocyte sedimentation ratio (ESR, $48 \mathrm{~mm} / \mathrm{h}$ ). Other laboratory tests were unremarkable, including autoantibodies and viral or microbial antibody titers (Table 1). Enhanced computed tomography and a gallium scintigraphy scan revealed no abnormal findings indicating any suspicion of pleuritis or peritonitis. Bone marrow examination demonstrated no abnormal cells. No ophthalmologic disorders, such as uveitis or iridocyclitis, were detected. After screening for the cause of his fever, a follow-up was performed, and the patient received antibiotics, ceftriaxone, minocycline, and meropenem, in that order. As these drugs did not improve his symptoms, antibiotic therapy was discontinued on day 9 of admission. However, he was spontaneously afebrile subsequently. The duration of his fever was a total of 24 days. Afterwards, he was followed up as an outpatient, with the cause of the previous fever indicated as unknown. He presented periodic fever of $38-39^{\circ} \mathrm{C}$ that lasted for 10 days, every 2-3 months following his discharge (Figure 1). His low total Gaslini diagnostic score [7] suggested a low risk for typical FMF, tumor necrosis factor receptor-associated periodic syndrome (TRAPS), and mevalonate kinase deficiency. Due to leukocytopenia during his febrile attacks, fluorodeoxyglucose-positron emission tomography was performed with the aim of ruling out intraperitoneal histiocytic necrotizing lymphadenitis (Kikuchi-Fujimoto disease). However, we could not detect any abnormal findings, such as FDG uptake at the mesenteric lymph nodes and joints, or abnormal masses. His blood test findings including leukocyte counts and inflammatory response during the intermittent period of fever were normal.

Subsequently, he sometimes complained of knee pains and presented skin rash during the attacks. On the basis of his periodic fever $\left(\geq 38^{\circ} \mathrm{C}\right)$ accompanied by elevated acute reactants such as CRP and SAA, we suspected an autoinflammatory syndrome and treated him with colchicine
$(0.5 \mathrm{mg} /$ day, $0.015 \mathrm{mg} / \mathrm{kg} /$ day). The patient was afebrile a day after colchicine administration. A gene analysis, including MEFV, TNFRSF1A, NLRP3, MVK, PLCG2, NLRC4, ADA2, TNFAIP3, TREX1, RNASEH2A, RNASEH2B, RNASEH2C, SAMHD1, ADAR, and IFIH1, showed the presence of a heterozygous $M E F V$ p.Ser503Cys exon 5 variant. Although the long febrile duration and leukocytopenia during the attacks were not typical symptoms of FMF, the presence of abdominal pain, the efficacy of treatment with colchicine, and the detection of heterozygous $M E F V$ p.Ser503Cys exon 5 variant suggested that he had an atypical FMF. He was initially treated with colchicine only during the febrile attacks. As complete elimination of the attacks was not gained, he was treated with colchicine regularly. Although a daily administration of $0.5 \mathrm{mg}(0.01 \mathrm{mg} / \mathrm{kg} /$ day $)$ colchicine did not prevent his febrile attacks, a daily dose of $1.5 \mathrm{mg}$ $(0.03 \mathrm{mg} / \mathrm{kg} /$ day $)$ colchicine achieved complete eradication of the attacks for more than one year.

\section{Discussion}

In this study, we described a Japanese boy with heterozygous $M E F V$ p.Ser503Cys exon 5 variant with periodic fever, which was improved by administration of colchicine. $M E F V$ heterozygous p.Ser503Cys exon 5 variant is reportedly found in approximately $2 \%$ of the Japanese population and in $0.6 \%-$ $1.9 \%$ of Japanese patients with FMF, a frequency higher than that observed in other races $[3,6,8]$. Moreover, FMF usually develops in patients with homozygous or compound heterozygous mutations in the $M E F V$ gene. Therefore, it is possible that the heterozygous $M E F V$ p.Ser503Cys exon 5 variant is one of the single nucleotide polymorphisms occurring in the Japanese population, and the patient's manifestations may have been incidental. However, in Japan, there are more patients with FMF, carrying heterozygous mutations in the $M E F V$ gene than those carrying homozygous mutations [3]. In addition, in a limited number of cases with a heterozygous $M E F V$ p.Ser503Cys exon 5 variant, the patients have been phenotypically diagnosed with atypical FMF (Table 2) [9-11]. It remains perplexing why patients with heterozygous $M E F V$ p.Ser503Cys exon 5 variant present atypical FMF manifestations. Some reports indicate that other factors, such as polymorphisms of human leukocyte antigen (HLA) or polymorphism in the gene associated with serum amyloid A, influence the risk of FMF expression $[12,13]$. Moreover, although we did not detect any additional gene mutations, a previous report describes a patient with a mutation in the NOD-like receptors family pyrin domain-containing 3 (NLRP3) gene, which is one of the molecules constituting the inflammasome, in addition with the heterozygous $M E F V$ p.Ser503Cys variant [11]. Additional mutations in genes involved in inflammation might be required for the development of symptoms in patients with heterozygous $M E F V$ p.Ser503Cys variant. However, a review of a greater number of heterozygous $M E F V$ p.Ser503Cys exon 5 variant cases is essential to speculate about the underlying pathophysiological mechanisms of atypical FMF and establish the best approach for its management. 
TABLE 1: Laboratory findings on admission.

\begin{tabular}{|c|c|c|c|c|c|c|}
\hline WBC & $5,460 / \mu \mathrm{L}$ & $\mathrm{Na}$ & $137 \mathrm{mEq} / \mathrm{L}$ & & Autoantibodies & Neg \\
\hline NEU & $78 \%$ & $\mathrm{~K}$ & $4.0 \mathrm{mEq} / \mathrm{L}$ & & & \\
\hline LYM & $15 \%$ & $\mathrm{Cl}$ & $98 \mathrm{mEq} / \mathrm{L}$ & & M. pneumoniae -IgM & Neg \\
\hline MONO & $7 \%$ & $\mathrm{Ca}$ & $9.1 \mathrm{mg} / \mathrm{dL}$ & & EBV VCA-IgG & $<10$ 1:n \\
\hline $\mathrm{RBC}$ & $498 \times 10^{4} / \mu \mathrm{L}$ & IP & $4.6 \mathrm{mg} / \mathrm{dL}$ & & EBV VCA-IgM & $<101: n$ \\
\hline $\mathrm{Hb}$ & $13.7 \mathrm{~g} / \mathrm{dL}$ & CRP & $3.30 \mathrm{mg} / \mathrm{dL}$ & & EBNA & $1601: n$ \\
\hline $\mathrm{Ht}$ & $40.9 \%$ & Procalcitonin & $0.58 \mathrm{ng} / \mathrm{mL}$ & $(<0.50)$ & CMV-IgG & Neg \\
\hline PLT & $27.9 \times 10^{4} / \mu \mathrm{L}$ & ESR (1 h) & $43 \mathrm{~mm}$ & & CMV-IgM & $\mathrm{Neg}$ \\
\hline RET & $1.2 \%$ & $\operatorname{IgG}$ & $1,270 \mathrm{mg} / \mathrm{dL}$ & & $\begin{array}{l}\text { Coxiella burnetii-IgG } \\
\text { (paired serum) }\end{array}$ & $<641: n$ \\
\hline AST & $31 \mathrm{IU} / \mathrm{L}$ & $\operatorname{Ig} A$ & $228 \mathrm{mg} / \mathrm{dL}$ & & & \\
\hline ALT & $25 \mathrm{IU} / \mathrm{L}$ & $\operatorname{IgM}$ & $120 \mathrm{mg} / \mathrm{dL}$ & & & \\
\hline$\gamma$-GTP & $14 \mathrm{IU} / \mathrm{L}$ & Ferritin & $57 \mathrm{ng} / \mathrm{mL}$ & $(21-282)$ & Urinalysis & \\
\hline $\mathrm{LDH}$ & $231 \mathrm{IU} / \mathrm{L}$ & sIL-2R & $498 \mathrm{U} / \mathrm{mL}$ & $(122-496)$ & $\mathrm{pH}$ & 6.5 \\
\hline $\mathrm{CK}$ & $23 \mathrm{IU} / \mathrm{L}$ & ANA & $<401: \mathrm{n}$ & & Red blood cell & Neg \\
\hline T-BIL & $0.3 \mathrm{mg} / \mathrm{dL}$ & CH50 & $64 \mathrm{U} / \mathrm{mL}$ & & White blood cell & Neg \\
\hline $\mathrm{TP}$ & $7.5 \mathrm{~g} / \mathrm{dL}$ & C3 & $205 \mathrm{mg} / \mathrm{dL}$ & & Protein & Neg \\
\hline Alb & $4.0 \mathrm{~g} / \mathrm{dL}$ & $\mathrm{C} 4$ & $51.7 \mathrm{mg} / \mathrm{dL}$ & & & \\
\hline BUN & $20.0 \mathrm{mg} / \mathrm{dL}$ & $\mathrm{RF}$ & $<15 \mathrm{U} / \mathrm{mL}$ & & & \\
\hline \multirow[t]{3}{*}{ CRE } & $0.83 \mathrm{mg} / \mathrm{dL}$ & MMP-3 & $30.1 \mathrm{ng} / \mathrm{dL}$ & $(37-121)$ & & \\
\hline & & SAA & $920.0 \mu \mathrm{g} / \mathrm{mL}$ & $(<8.0)$ & & \\
\hline & & B-2 microglobulin & $2.8 \mathrm{mg} / \mathrm{L}$ & $(0.0-2.0)$ & & \\
\hline
\end{tabular}

sIL-2R, soluble interleukin-2 receptor; ANA, antinuclear antibody; RF, rheumatoid factor; MMP-3, matrix metalloproteinase-3; SAA, serum amyloid A; M. pneumoniae, Mycoplasma pneumoniae; EBV, Epstein-Barr virus; VCA, virus capsid antigen; EBNA, EBV nuclear antigen; CMV, cytomegalovirus; Neg, negative; (), normal range.

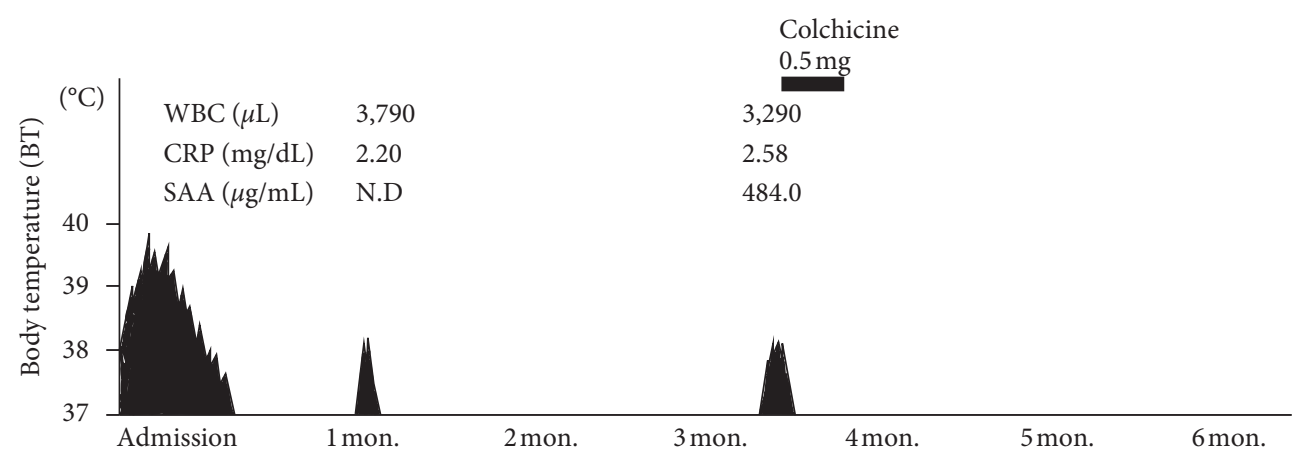

(a)

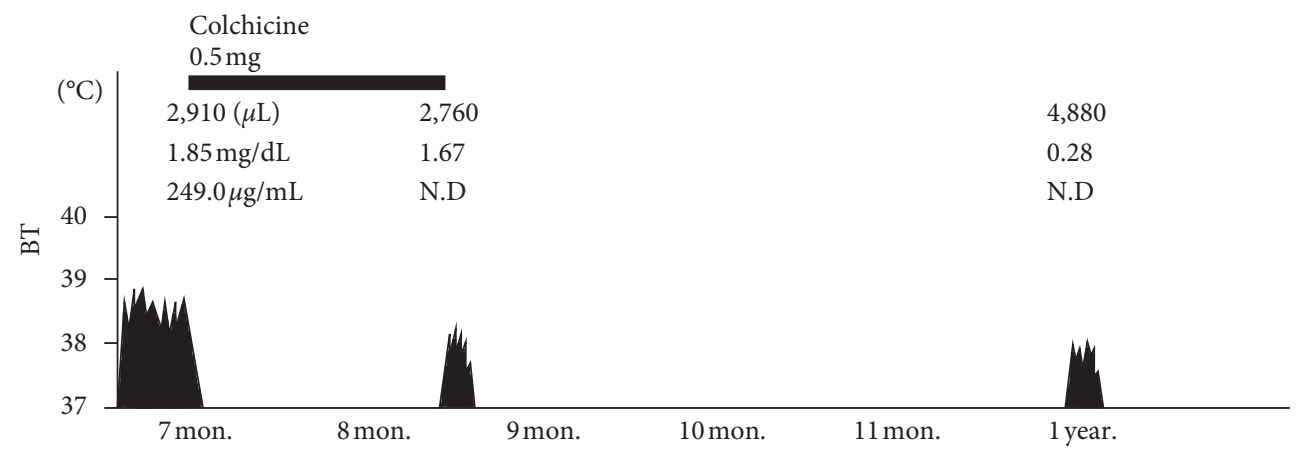

(b)

FIGURE 1: Continued. 


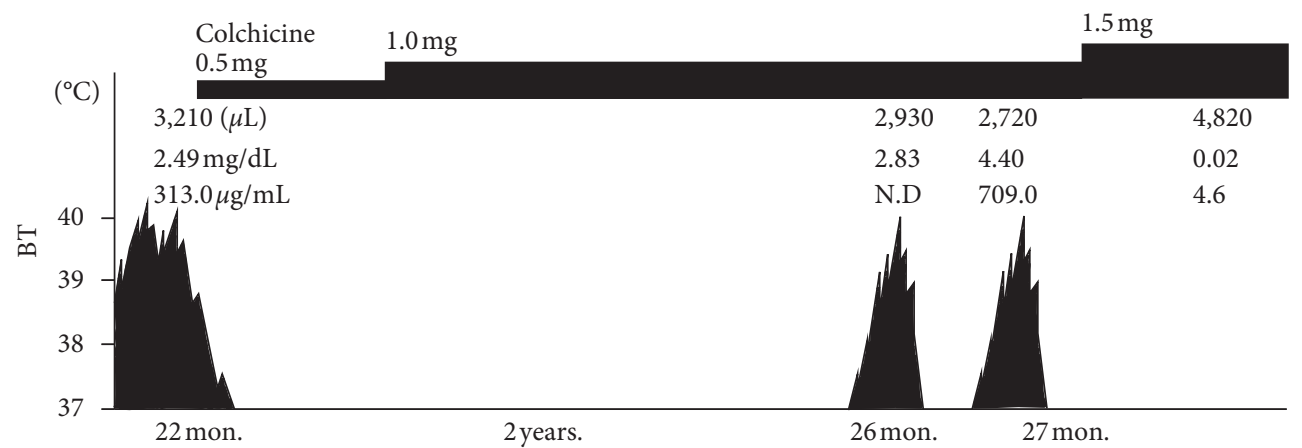

(c)

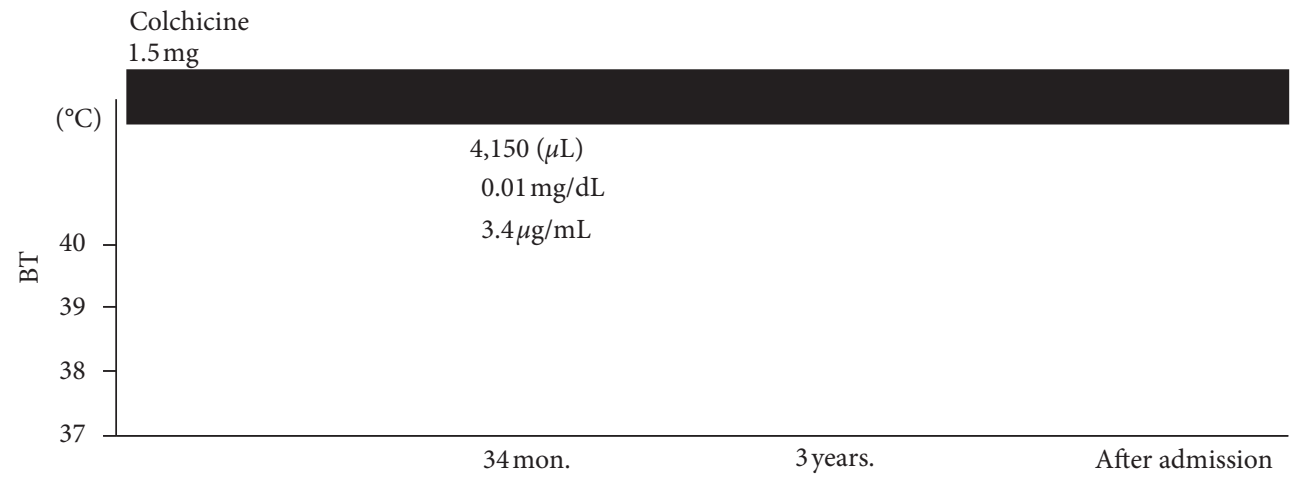

(d)

FIGURE 1: The clinical course. Horizontal axis shows months after the patient's admission, and vertical axis shows patient's body temperature (BT, notated Celsius temperature). Jagged black area means the duration and the height of patient's fever. WBC, white blood cells; CRP, Creactive protein; SAA, serum amyloid A; N.D, no data.

TABLE 2: Profile of previously reported FMF patients with $M E F V$ p.Ser503Cys exon 5 variants.

\begin{tabular}{|c|c|c|c|c|c|c|c|c|c|c|}
\hline \multirow{2}{*}{ Patient (Ref.) } & \multirow{2}{*}{ Age $(y r) /$ sex } & \multirow{2}{*}{$M E F V$} & \multirow{2}{*}{ FMF } & \multicolumn{2}{|c|}{ Febrile attack } & \multicolumn{5}{|c|}{ Other symptoms of FMF } \\
\hline & & & & Frequency (mo) & Duration (day) & $\mathrm{Ab}$. & Th. & Arth. & Head. & Response to colchicine \\
\hline [9] & $38 /$ male & E148Q/S503C & Atyp. & $<1$ & 14 & + & + & - & + & + \\
\hline [10] & $47 /$ male & S503C/- & Atyp. & 2 & 3 & + & - & + & + & + \\
\hline$[11]$ & $65 /$ male & $\mathrm{S} 503 \mathrm{C} /-$ & Atyp. & NA & NA & + & - & + & NA & + \\
\hline Our case & $11 /$ male & S503C/- & Atyp. & $1-3$ & 10 & + & - & - & + & + \\
\hline
\end{tabular}

Ab., abdominal pain; Arth., arthralgia; Atyp., atypical FMF; FMF, familial Mediterranean fever; Head., headache; mo, month; NA, not available; Ref.; reference number; Th., thoracic pain; Typ., typical FMF; yr, year.

In our case, the patient developed periodic fever after age 10 and demonstrated fever, accompanied with aphthous stomatitis and slight abdominal pain. Although blood biochemistry tests revealed the presence of high acute phase reactants and elevated erythrocyte sedimentation ratio, his complete blood counts often revealed leukocytopenia during the fever bouts. Moreover, treatment with increasing doses of colchicine improved his periodic fever and accompanying symptoms. Although the detailed pathophysiology is unknown, previous reports describe that similar to our case, atypical FMF patients demonstrated leukopenia during febrile attacks $[14,15]$. However, these patients were not reported to have the $M E F V$ exon5 p.Ser503Cys variant, indicating that leukocytopenia during febrile attack in atypical FMF is not limited to the $M E F V$ exon5 p.Ser503Cys variant.
According to the Gaslini diagnostic score, the presence of aphthous stomatitis during febrile episodes and the high age of onset are the factors that do not positively indicate typical FMF, TRAPS, or mevalonate kinase deficiency [7]. The presence of aphthous stomatitis especially made it difficult to distinguish the disease from other autoinflammatory and autoimmune diseases, such as PFAPA syndrome and Behcet's disease (BD). Without diseasespecific tests such as colonoscopy and/or HLA analysis, it is hard to rule out $\mathrm{BD}$, and there is a possibility that the patient was in an early stage of BD. However, in our case, though aphthous stomatitis was present, the skin, ocular, and vulvar symptoms, which are characteristic of $\mathrm{BD}$, were not observed. In addition, no vascular or central nervous system lesions were found, and laboratory tests revealed no leukocytosis. Hence, we concluded that it was unlikely that this 
patient had BD. Recently, there have been reports on a group of diseases exhibiting a BD-like phenotype. Perazzio et al. reported that a $\mathrm{BD}$-like phenotype is at the crossroads of autoimmune and autoinflammatory syndromes and is associated with a variety of congenital immune disorders including FMF [16]. Since BD and FMF are overlapping disease groups, it could be difficult to make a strict distinction between them.

However, the presence of abdominal pain, which is suspicious of nonlocalized peritonitis, and the efficacy of colchicine in the reduction of fever were factors that suggested atypical FMF. To diagnose FMF, either the Tel Hashomer criteria or the criteria of the diagnostic tool for FMF, proposed by the Ministry of Health, Japan, are referred [17-19]. According to Tel Hashomer diagnostic criteria, in this case, his symptoms were consistent with fever as a major criterion and the effectiveness of colchicine as a minor criterion. However, he could not be regarded as a typical FMF patient as he had febrile episodes lasting longer than three days and no accompanying symptoms such as pleuritis or arthritis. Notably, in Japan, the number of patients with atypical FMF exceeds those with typical FMF with $M E F V$ exon 10 mutation, with symptoms such as peritonitis and pleuritis, which are common in typical FMF patients of other ethnic groups. Therefore, in addition to Tel Hashomer criteria, the diagnostic criteria for FMF proposed by the Ministry of Health, Japan, are also used [19], which propose that fever with an elevation of serum acute phase reactants is considered an essential criterion, and the presence of symptoms due to serositis such as pleuritis or the effectiveness of colchicine are considered auxiliary criteria for a clinical diagnosis of FMF. In addition, patients with $M E F V$ exon 10 mutations are diagnosed with typical FMF, and patients with mutations in other regions of the $M E F V$ gene are diagnosed with atypical FMF. In the present case, the patient demonstrated prolonged fever for more than four days, accompanied by elevated serum acute phase reactants, abdominal pain, headache during febrile episodes, and fever remission upon treatment with colchicine. The $M E F V$ variant amino acid substitution and his clinical manifestations suggest the patient's diagnosis as an atypical FMF according to diagnostic criteria prescribed by the Ministry of Health, Japan.

Our patient had frequent complaints of headache, which is not included in the symptoms of FMF. We did not perform further evaluation of his cerebrospinal fluid; however, a study reported an atypical FMF patient with compound heterozygous mutations in exons 2 and 5 (E148Q/S503C) in MEFV who experienced recurrent headache due to chronic aseptic meningitis [9]. Although it is difficult to strongly suggest from such a small number of cases, it is possible that headache due to aseptic meningitis is one of the complications in atypical FMF, and atypical FMF could be a differential diagnosis in periodic fever patients with headaches.

In conclusion, here, we reported a Japanese boy with heterozygous $M E F V$ p.Ser503Cys exon 5 variant with periodic fever that was improved by treatment with colchicine and remains afebrile to date. Since there have been few reports on patients with this variant, little is known about its genetic and phenotypic role. When such cases are encountered, therefore, a consideration should be given for an atypical FMF differential diagnosis. Furthermore, for these cases, we speculate that it is worth conducting an $M E F V$ gene analysis and testing colchicine treatment for potential relief. Early diagnosis and therapeutic intervention for suspected FMF patients is pivotal to avoid the accumulation of SAA and especially to understand the clinical characteristics and therapeutic strategies for suspected patients of atypical FMF with heterozygous $M E F V$ p.Ser503Cys exon 5 variant.

\section{Ethical Approval}

The present study adhered to the Declaration of Helsinki.

\section{Consent}

The patient's guardians signed an informed consent to publish this report.

\section{Conflicts of Interest}

The authors declare that they have no conflicts of interest.

\section{Acknowledgments}

The authors would like to thank Kazusa DNA Research Institute for the patient's genetic analysis.

\section{References}

[1] P. Wekell, A. Karlsson, S. Berg, and A. Fasth, "Review of autoinflammatory diseases, with a special focus on periodic fever, aphthous stomatitis, pharyngitis and cervical adenitis syndrome," Acta Paediatrica, vol. 105, no. 10, pp. 1140-1151, 2016.

[2] R. Heilig and P. Broz, "Function and mechanism of the pyrin inflammasome," European Journal of Immunology, vol. 48, no. 2, pp. 230-238, 2018.

[3] K. Migita, K. Agematsu, M. Yazaki et al., "Familial mediterranean fever," Medicine, vol. 93, no. 3, pp. 158-164, 2014.

[4] J. G. Ryan, S. L. Masters, and M. G. Booty, "Clinical features and functional significance of the P369S/R408Q variant in pyrin, the familial mediterranean fever protein," Annals of the Rheumatic Diseases, vol. 69, no. 7, pp. 1383-1388, 2010.

[5] S. Padeh, A. Livneh, E. Pras et al., "Familial Mediterranean Fever in the first two years of life: a unique phenotype of disease in evolution," The Journal of Pediatrics, vol. 156, no. 6, pp. 985-989, 2010.

[6] D. Kishida, A. Nakamura, and M. Yazaki, "Genotype-phenotype correlation in Japanese patients with familial mediterranean fever: differences in genotype and clinical features between Japanese and mediterranean populations," Arthritis Research \& Therapy, vol. 16, no. 5, p. 439, 2014.

[7] M. Gattorno, M. P. Sormani, A. D’Osualdo et al., "A diagnostic score for molecular analysis of hereditary autoinflammatory syndromes with periodic fever in children," Arthritis \& Rheumatism, vol. 58, no. 6, pp. 1823-1832, 2008.

[8] K. Fujikura, "Global epidemiology of familial mediterranean fever mutations using population exome sequences," 
Molecular Genetics \& Genomic Medicine, vol. 3, no. 4, pp. 272-282, 2015.

[9] M. Sugie, T. Ouchi, D. Kishida, and S. Yasaki, "Atypical type of familial mediterranean fever: an underdiagnosed cause of chronic aseptic meningitis," Neurology and Clinical Neuroscience, vol. 6, no. 6, pp. 191-193, 2018.

[10] K. Kimura, M. Mizooka, K. Migita et al., "Five cases of familial mediterranean fever in Japan: the relationship with $M E F V$ mutations," Internal Medicine, vol. 57, no. 16, pp. 2425-2429, 2018.

[11] K. Fujimoto, Y. Hidaka, T. Koga et al., "Clinical and genetic analysis of 22 Japanese patients with familial mediterranean fever: an examination of $M E F V$ and 10 other genes related to autoinflammatory syndromes," Internal Medicine, vol. 59, no. 11, pp. 1373-1378, 2020.

[12] M. Yasunami, H. Nakamura, and K. Agematsu, "Identification of disease-promoting HLA class I and protective class II modifiers in Japanese patients with familial mediterranean fever," PLoS One, vol. 10, no. 5, Article ID e0125938, 2015.

[13] K. Migita, K. Agematsu, and J. Masumoto, "The contribution of SAA1 polymorphisms to familial mediterranean fever susceptibility in the Japanese population," PLoS One, vol. 8, no. 2, Article ID e55227, 2013.

[14] I. Beyitler and S. Kavukcu, "A case of familial mediterranean fever having intermittent leukopenia," Journal of Pediatric Hematology/Oncology, vol. 40, no. 2, pp. e111-e112, 2018.

[15] O. Sakallioglu, "Leucopenia resoluted with colchicine in familial mediterranean fever," Journal of Pediatric Hematology/ Oncology, vol. 34, no. 2, p. 162, 2012.

[16] S. F. Perazzio, E. J. Allenspach, and K. K. Eklund, "Behçet disease $(\mathrm{BD})$ and $\mathrm{BD}$-like clinical phenotypes: NF- $\kappa \mathrm{B}$ pathway in mucosal ulcerating diseases," Scandinavian Journal of Immunology, vol. 92, no. 5, Article ID e12973, 2020.

[17] A. Livneh, P. Langevitz, D. Zemer et al., "Criteria for the diagnosis of familial mediterranean fever," Arthritis \& Rheumatism, vol. 40, no. 10, pp. 1879-1885, 1997.

[18] A. Tsuchiya-Suzuki, M. Yazaki, A. Nakamura et al., "Clinical and genetic features of familial mediterranean fever in Japan," The Journal of Rheumatology, vol. 36, no. 8, pp. 1671-1676, 2009.

[19] K. Migita, T. Asano, S. Sato, T. Koga, Y. Fujita, and A. Kawakami, "Familial Mediterranean fever: overview of pathogenesis, clinical features and management," Immunological Medicine, vol. 41, no. 2, pp. 55-61, 2018. 